\title{
Bestimmung des komplexen Brechungsindex eines Plasmas mittels des Ausbreitungsverfahrens beim Auftreten reflektierter Wellen
}

\author{
W. MusChLER \\ Max-Planck-Institut für Aeronomie, Abteilung Weltraumphysik, Lindau (Harz) \\ (Z. Naturforsch. 25 a, 482-487 [1970]; eingegangen am 17. Dezember 1969)
}

\begin{abstract}
In a preceding paper a propagation method has been described that allows simultaneous determination of electron concentration and electron collision frequency in a slowly varying plasmausing its complex refractive index. A regular progressive damped wave had been assumed. In this paper the treatment is extended to the case that the original wave is superimposed by a reflected one. The calculations show that for distinct points in the direction of wave propagation the determination of both modulus and argument of the complex refractive index is again possible. The discrete sequence of measuring points does not restrict the local resolution of the method.
\end{abstract}

\section{§ 1. Einleitung}

In einer vorangegangenen Arbeit ${ }^{1}$ wurde ein Verfahren zur gleichzeitigen Bestimmung von Elektronenkonzentration und Elektronenstoßzahl in einem ionisierten Gas beschrieben. Das Grundprinzip geht auf die Ermittlung beider Bestimmungselemente $|n|$ (Betrag) und $\varphi$ (Argument) des komplexen Brechungsindex zurück: Die $E$ - und die $H$-Komponente der zur Messung dienenden elektromagnetischen Welle liefern über ihr Amplitudenverhältnis den Wert $|n|$, über ihre Phasendifferenz den Wert $\varphi$. Die Überlegungen galten für ein homogenes isotropes oder für ein örtlich langsam veränderliches Medium. Demzufolge konnte eine gewöhnliche fortschreitende, gedämpfte Welle angenommen werden.

Bei der Anwendung des Verfahrens kann nicht ausgeschlossen werden, daß die fortschreitende Welle von einer reflektierten überlagert wird. Die Bedeutung solcher Reflexionen läßt sich am Sonderfall stehender Wellen leicht erkennen: Die Komponenten der elektromagnetischen Welle sind örtlich wie zeitlich phasenverschoben, das Amplitudenverhältnis wird extrem ortsabhängig. Betrachtungen über den Einfluß reflektierter Wellen auf das Meßverfahren sind daher angebracht.

Bei den folgenden Überlegungen wird wieder von Theorie und Gegebenheiten der Ionosphärenphysik ausgegangen.

Sonderdruckanforderungen an Dr. W. Muschler, MaxPlanck-Institut für Aeronomie, Abteilung Weltraumphysik, D-3411 Lindau (Harz), Postfach 70.

1 W. Muschler, Z. Naturforsch. 25 a, 106 [1970].

\section{§ 2. Kriterien für das Auftreten reflektierter Wellen}

Reflexionen treten an den Grenzflächen von Medien mit unterschiedlichem Brechungsindex auf. Liegt ein Medium mit veränderlichem Brechungsindex vor, so entstehen in ihm solange keine feststellbaren Reflexionen, wie die Änderungen ,genügend langsam" vor sich gehen. Für ein solches Medium war die Wellenausbreitung durch die WKBLösungen der Wellengleichung zu beschreiben. Die Bedingung für die Gültigkeit der WKB-Lösung lautet dabei ${ }^{2}$ :

$$
B_{\mathrm{WKB}} \equiv \frac{1}{k^{2}} \frac{3}{4}\left(\frac{1}{n^{2}} \cdot \frac{\mathrm{d} n}{\mathrm{~d} z}\right)^{2}-\frac{1}{2} \cdot \frac{1}{n^{3}} \cdot \frac{\mathrm{d}^{2} n}{\mathrm{~d} z^{2}} \mid \ll 1,
$$

$k=$ Wellenzahl, $n=$ komplexer Brechungsindex, $z=$ Ausbreitungsrichtung.

Ein ähnliches Kriterium für die Entstehung von Reflexionen ist der Gradienten-Reflexionskoeffizient (s. Anm. ${ }^{3}$ ) :

$$
\varrho_{\mathrm{G}}=\frac{1}{4 k \mu^{2}} \cdot \frac{\mathrm{d} \mu}{\mathrm{d} z},
$$

$\mu=$ Realteil des Brechungsindex.

Beide Kriterien sind für die in ${ }^{1}$ benutzte ModellIonosphäre berechnet und in ${ }^{1}$ bzw. Abb. 1 dargestellt.

Die Abbildungen lassen den erwarteten Sachverhalt erkennen: Bei niedrigen Frequenzen sind Re-

${ }^{2}$ K. G. Budden, Radio Waves in the Ionosphere, Cambridge University Press 1966, S. 133.

3 J. A. Ratcliffe, The Magneto-Ionic Theory, Cambridge University Press 1962, S. 175. 


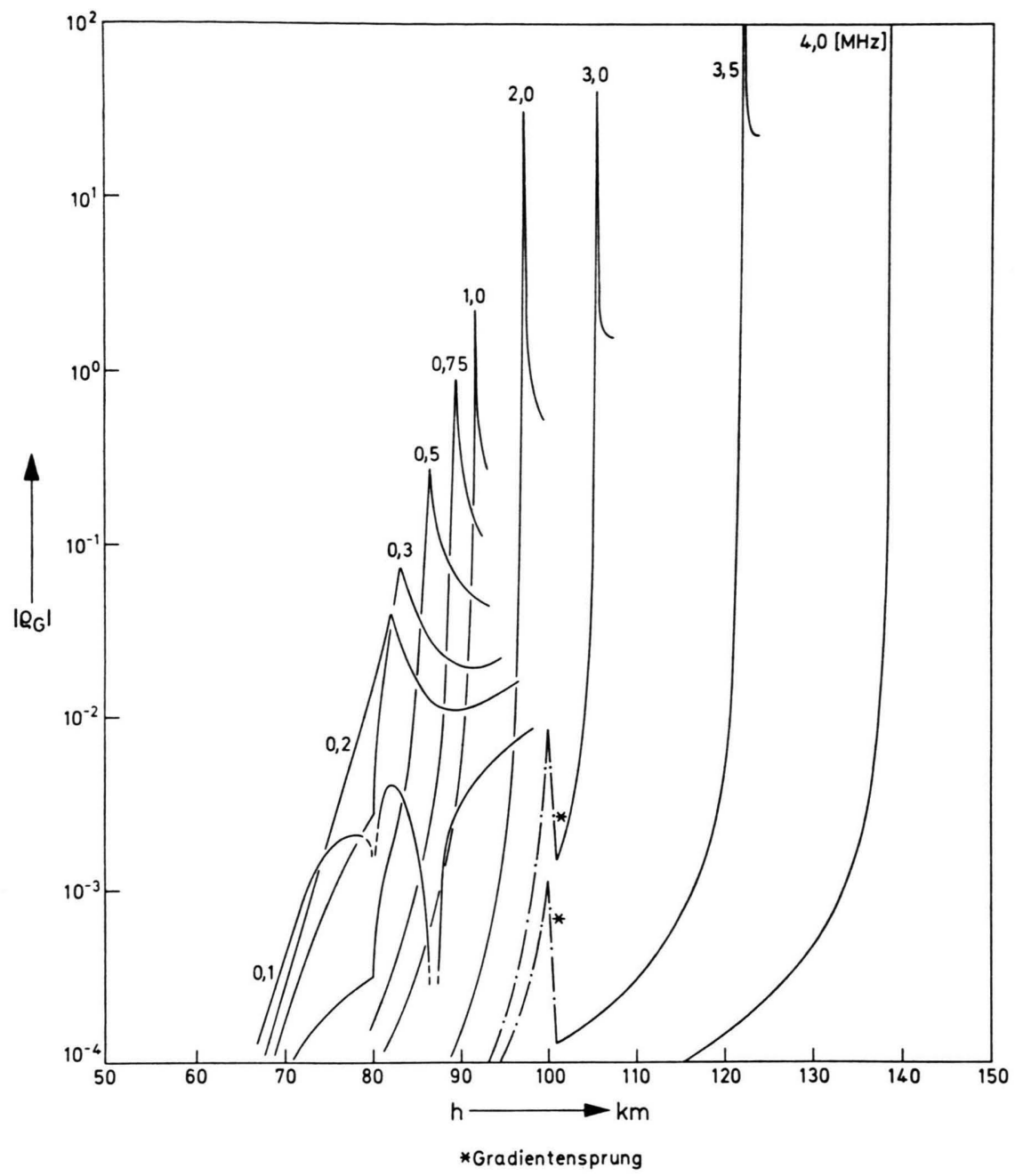

Abb. 1. Größenordnung des Gradienten-Reflexionskoeffizienten für die in 1 charakterisierte Modell-Ionosphäre $\left(\left|\varrho_{\mathrm{G}}\right|>1\right.$ sei mit $\left|\varrho_{\mathrm{G}}\right|=1$ physikalisch gleichbedeutend).

flexionen praktisch vernachlässigbar. Mit wachsender Frequenz fallen sie mehr und mehr ins Gewicht, bis sich schließlich die scharf lokalisierte Totalreflexion einstellt.

\section{§ 3. Partielle stehende Wellen als Interferenz- erscheinung hin- und rücklaufender Wellen im absorbierenden Plasma}

Wir interessieren uns für denjenigen Bereich des Plasmas, innerhalb dessen die Amplituden der hinund rücklaufenden Welle durch die WKB-Lösungen beschrieben werden können. Dort gilt für die hinlaufende ebene Welle ${ }^{1}$ :

$$
E_{1} \cong \frac{A}{\sqrt{n}} \exp \left\{-i k \int_{0}^{r} n \mathrm{~d} z+i \omega t\right\},
$$

$$
H_{1} Z_{0} \cong A \sqrt{n} \exp \left\{-i k \int_{0}^{z} n \mathrm{~d} z+i \omega t\right\} .
$$

Die rücklaufende Welle wird folgendermaßen beschrieben ${ }^{1,4}$ :

$$
\begin{array}{r}
E_{2} \cong \frac{A R}{\sqrt{n}} \exp \left\{i k \int_{0}^{z} n \mathrm{~d} z+i \omega t\right\}, \\
H_{2} Z_{0} \cong-A R \sqrt{n} \exp \left\{i k \int_{0}^{z} n \mathrm{~d} z+i \omega t\right\} .
\end{array}
$$

$A$ und $A R$ stellen die Amplituden der hin- bzw. rücklaufenden Welle an der Stelle $z=0$ für $n=1$ dar. Der komplexe Reflexionskoeffizient soll hier mit seinem Betrag $|R|$ genommen werden, da es bei den weiteren Betrachtungen nicht auf die anfängliche Phasenlage der rückkehrenden Welle ankommt.

${ }^{4}$ K. G. Budden, l. c. ${ }^{2}$, S. 136. 
$|R|$ beinhaltet so die relative Amplitudenabnahme $R_{0}$ der Welle infolge Absorption auf dem Wege von $z=0$ zum Reflexionsniveau $z=z_{0}$ und zurück zum Ort $z=0$. Soll $|R|$ außerdem ein mehr oder weniger gutes Reflexionsvermögen $\varrho$ im Reflexionsbereich zum Ausdruck bringen, so ist zu schreiben:

$$
|R|=R_{0} \cdot \varrho \text {, wobei } R_{0}=\exp \left\{-2 k \int_{0}^{z_{0}} \chi \mathrm{d} z\right\} \text {. }
$$

$\varrho$ und $z_{0}$ lassen sich beispielsweise aus $\varrho_{G}=\varrho_{G}(z)$ (Abb. 1) abschätzen.

Nach den Umformungen

$$
E_{1}=\frac{A}{V|n|} \exp \left\{i \omega t-i k \int_{0}^{z} \mu \mathrm{d} z+i \varphi / 2\right\}
$$

und $\cdot \exp \left\{-k \int_{0}^{z} \chi \mathrm{d} z\right\}$

$$
\begin{array}{r}
E_{2}=\frac{A|R|}{V|\bar{n}|} \exp \left\{i \omega t+i k \int_{0}^{z} \mu \mathrm{d} z+i \varphi / 2\right\} \\
\cdot \exp \left\{k \int_{0}^{z} \chi \mathrm{d} z\right\}
\end{array}
$$

und nach Einführung der Größen

$$
\sigma=k \int_{0}^{z} \mu \mathrm{d} z, \quad \alpha=k \int_{0}^{z} \chi \mathrm{d} z, \quad \gamma=\varphi / 2
$$

werden die Realteile von $E_{1}$ und $E_{2}$ gebildet und diese überlagert:

$$
\begin{aligned}
\Re(E) & =\Re\left(E_{1}\right)+\Re\left(E_{2}\right) \\
& =(A / V|n|) e^{-\alpha \cdot \cos (\omega t-\sigma-\gamma)} \\
& +\frac{A}{V|n|}|R| e^{\alpha} \cdot \cos (\omega t+\sigma+\gamma) .
\end{aligned}
$$

Analog erhält man für die resultierende magnetische Komponente

$$
\begin{aligned}
\Re\left(H Z_{0}\right) & =\Re\left(H_{1} Z_{0}\right)+\Re\left(H_{2} Z_{0}\right) \\
& =A V|n| e^{-\alpha} \cdot \cos (\omega t-\sigma-\gamma) \\
& -A|R| V|n| e^{\alpha} \cdot \cos (\omega t+\sigma-\gamma) .
\end{aligned}
$$

Wir verkürzen weiter mit Hilfe der Definitionen

$$
\begin{aligned}
& A_{1}=\frac{A}{V|n|} e^{-\alpha}, \quad A_{2}=\frac{A}{V|n|}|R| e^{\alpha}, \quad(3.10), \\
& \qquad B_{1}=A \sqrt{|n|} e^{-\alpha}, B_{2}=A|R| V \mid \overline{n \mid} e^{\alpha} \\
& \text { und } \quad d=\frac{A_{2}}{A_{1}}=\frac{B_{2}}{B_{1}}=|R| e^{2 \alpha} \\
& \text { Dabei gilt: } \quad B_{1,2}=|n| \cdot A_{1,2} .
\end{aligned}
$$

Man gelangt so zu den Gleichungen

$$
\begin{aligned}
& \Re(E) \equiv E_{\mathrm{s}}=A_{1}[\cos (\omega t-\sigma+\gamma)+d \cdot \cos (\omega t+\sigma+\gamma)], \\
& \Re\left(H Z_{0}\right) \equiv H_{\mathrm{s}} Z_{0}=B_{1}[\cos (\omega t-\sigma-\gamma)-d \cdot \cos (\omega t+\sigma-\gamma)] .
\end{aligned}
$$

Durch trigonometrische Umformung ergibt sich dann:

$$
\begin{aligned}
\frac{E_{\mathrm{s}}}{A_{1}} & =(1+d) \cos (\omega t+\gamma) \cos \sigma+(1-d) \sin (\omega t+\gamma) \sin \sigma, \\
\frac{H_{\mathrm{s}} Z_{0}}{B_{1}} & =(1-d) \cos (\omega t-\gamma) \cos \sigma+(1+d) \sin (\omega t-\gamma) \sin \sigma .
\end{aligned}
$$

Diese Gleichungen stellen eine partielle stehende Welle dar. $d$ ist hierbei - wie $A_{1}$ und $B_{1}$ - ortsabhängig. Die (zeitunabhängigen) Enveloppen über $\operatorname{der} \sigma$-Achse lassen sich ermitteln zu

$$
\left(\frac{E_{\mathrm{s}}}{A_{1}}\right)_{\mathrm{e}}=\frac{\left(E_{\mathrm{s}}\right)_{\mathrm{e}}}{A_{1}}= \pm \sqrt{1+d^{2}+2 d \cos 2 \sigma}
$$

bzw. $\left(\frac{H_{\mathrm{S}} Z_{0}}{B_{1}}\right)_{\mathrm{e}}=\frac{\left(H_{\mathrm{s}} Z_{0}\right)_{\mathrm{e}}}{B_{1}}= \pm \sqrt{1+d^{2}-2 d \cos 2 \sigma}$.

Sie sind in Abb. 2 für die vereinfachenden Annahmen $n=$ konstant (außerhalb der Reflexionszone) und $\varrho=1$ dargestellt; die Produkte $k \mu$ und $k \chi$ wurden willkürlich gewählt. Durch Multiplikation der Gleichungen (3.20) und (3.21) mit $B_{1}$ gewinnt man die Enveloppen der Größen $E_{\mathrm{s}}|n|$ und $H_{\mathrm{s}} Z_{0}$. Diese sind in Abb. 3 unter den Annahmen der Abb. 2 ver-

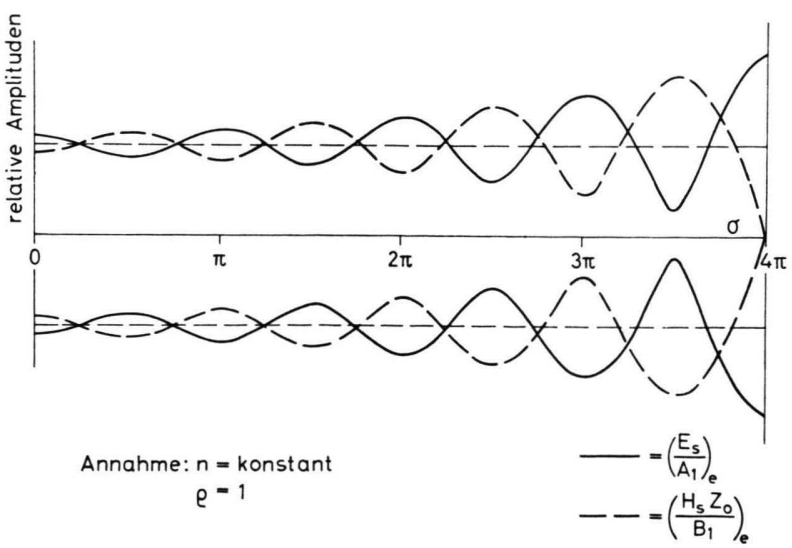

Abb. 2. Partielle stehende Welle: Darstellung der Enveloppen der resultierenden elektrischen und magnetischen Feldstärke $E_{\mathrm{s}}$ bzw. $H_{\mathrm{s}} Z_{0}$, bezogen auf die Amplitude $A_{1}$ bzw. $B_{1}$ der ursprünglichen (hinlaufenden) Welle am Ort $z$ $(\chi / \mu=\tan \varphi=0,09)$. 
anschaulicht. Durch die ortsfesten Maxima und Minima der partiellen stehenden Welle bewegt sich die verbleibende fortschreitende Welle mit atmenden Amplituden.

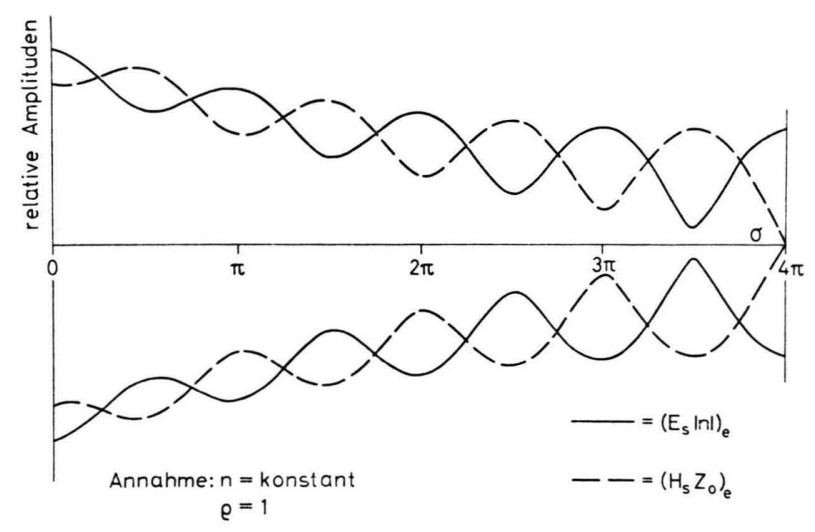

Abb. 3. Partielle stehende Welle: Darstellung der Enveloppen der resultierenden elektrischen und magnetischen Feldstärke in der Form $E_{\mathrm{S}}|n|$ und $H_{\mathrm{S}} Z_{0}(\chi / \mu=\tan \varphi=0,09)$.

\section{§ 4. Anwendung des KB-Verfahrens beim Auftreten partieller stehender Wellen}

\subsection{Ermittlung des Betrages des komplexen Brechungsindex}

Im reflexionsfreien Fall war $|n|$ aus dem Amplitudenverhältnis beider Komponenten der elektromagnetischen Welle zu ermitteln. Treten partielle stehende Wellen auf, und betrachtet man die Enveloppen beider Komponenten, so ist deren Verhältnis stark ortsabhängig (Abb. 3). An ihrer Stelle seien daher diejenigen monotonen Kurven betrachtet, auf welche die Maxima bzw. Minima (Täler) der Enveloppe zu liegen kommen. Diese Kurven seien als „Extremalkurven“ bezeichnet.

Nach dem üblichen Verfahren der Differentialrechnung gewinnt man aus den Gln. (3.20) und (3.21) die $\sigma$-Werte der Extrema von $\left(E_{\mathrm{s}}\right)_{\mathrm{e}}$ und $\left(H_{\mathrm{s}} Z_{0}\right)_{\text {e }}$ (hierbei ist die Ortsabhängigkeit von $A_{1}$, $B_{1}$ und $d$ zu beachten). Es gilt:

$$
\sin 2 \sigma_{\mathrm{E}}=\frac{d^{2}-1}{2 d} \tan \varphi=s
$$

und

$$
\sin 2 \sigma_{\mathrm{H}}=-s .
$$

Durch Einsetzen in die Gln. (3.20) und (3.21) ergeben sich die gesuchten Extremalkurven zu

$$
\left(E_{\mathrm{s}}\right)_{\mathrm{e}}^{\mathrm{extr}}= \pm A_{1} \sqrt{1+d^{2} \pm 2 d \sqrt{1-s^{2}}}
$$

und

$$
\left(H_{\mathrm{s}} Z_{0}\right)_{\mathrm{e}}^{\mathrm{extr}}= \pm B_{1} \sqrt{1+d^{2} \pm 2 d \sqrt{1-s^{2}} .}
$$

Sie sind über $d=d(\sigma)$ Funktionen von $\sigma$. Das positive Vorzeichen der inneren Quadratwurzel entspricht in beiden Gleichungen der Kurve der Maxima, das negative Vorzeichen der Kurve der Minima.

Division von (4.3) und (4.4) liefert zusammen mit (3.15)

$$
\frac{\left(H_{\mathrm{S}} Z_{0}\right)_{\mathrm{e}}^{\text {extr }}}{\left(E_{\mathrm{S}}\right)_{\mathrm{e}}^{\text {extr }}}=\frac{B_{1}}{A_{1}}=|n| .
$$

Bei der meßtechnischen Anwendung sind die Extremalkurven durch die im Abstand einer halben Wellenlänge folgenden Maxima bzw. Minima der Enveloppen punktweise gegeben. Die gegenseitige Versetzung der Maxima und Minima um eine Viertelwellenlänge verbessert die Meßgenauigkeit.

\subsection{Die Lage der Extrema der Enveloppe}

Wie die Gln. (4.1) und (4.2) zeigen, fallen $\sigma_{\mathrm{F}}$ und $\sigma_{\mathrm{H}}$ durch iden Einfluß der Absorption nicht mehr zusammen. Die Maxima beider Komponenten verschieben sich entgegengesetzt wie deren Minima. Gegenüber dem absorptionsfreien Fall mit $\tan \varphi=0$ und $\sigma_{\mathrm{E}}=\sigma_{\mathrm{H}}=0, \frac{\pi}{2}, \pi, \ldots$ beträgt die Versetzung

$$
\Delta \sigma= \pm \frac{1}{2} \arcsin |s|,
$$

wobei das positive Vorzeichen für die Minima, das negative für die Maxima gilt. Abb. 3 läßt die Verschiebungen im Bereich kleiner $\sigma$-Werte deutlich werden.

Für ein langsam veränderliches Medium kann $\varphi$ in (4.1) abschnittweise als konstant angesehen werden. Wenn weiterhin von Fällen allzu hoher Absorption (große $\varphi$ - und kleine $d$-Werte) abgesehen werden darf, so ist auch die Ortsabhängigkeit von $d$ für das relativ schmale Intervall von $\sigma_{\mathrm{E}}$ bis $\sigma_{\mathrm{H}}$ vernachlässigbar. Dann kann für zusammengehörige $\sigma_{\mathrm{E}}$ und $\sigma_{\mathrm{H}}$-Werte ein gleich großer Absolutwert von $s$ gelten, und es ergibt sich nach (4.6) Symmetrie von $\sigma_{\mathrm{E}}$ und $\sigma_{\mathrm{H}}$ gegenüber dem Zentralwert $0, \frac{\pi}{2}, \pi, \ldots$.

\subsection{Bestimmung des Argumentes des komplexen Brechungsindex}

Im reflexionsfreien Fall konnte $\varphi$ in einfacher Weise über die Phasendifferenz zwischen den beiden Komponenten der elektromagnetischen Welle erhalten werden. Beim Auftreten reflektierter Wellen wird erheblicher Einfluß auf einen solchen Meßvorgang erwartet. 
Zur Untersuchung dieses Einflusses wird von den Gln. (3.18) und (3.19) ausgegangen und nach denjenigen Zeiten $t_{\mathrm{E}}$ und $t_{\mathrm{H}}$ bei konstantem Ort $\sigma$ gefragt, die maximale $E_{\mathrm{s}}$ - bzw. $H_{\mathrm{s}} Z_{0}$-Amplituden liefern. Nach bekanntem Vorgehen gewinnt man die Gleichungen

$$
\tan \left(\omega t_{\mathrm{E}}+\gamma\right)=\frac{1-d}{1+d} \tan \sigma
$$

und

$$
\tan \left(\omega t_{\mathrm{H}}-\gamma\right)=\frac{1+d}{1-d} \tan \sigma .
$$

Setzt man die rechten Seiten von (4.7) und (4.8) gleich $\tau_{1}$ bzw. $\tau_{2}$, so folgt weiter

$$
\omega t_{\mathrm{H}}-\omega t_{\mathrm{E}}=\varphi-\arctan \tau_{1}+\arctan \tau_{2} .
$$

Der Phasenunterschied $\omega t_{\mathrm{H}}-\omega t_{\mathrm{E}} \equiv \delta$ hängt jetzt nicht mehr allein von $\varphi \mathrm{ab}$, sondern ist durch Zusatzterme stark ortsabhängig geworden. Abb. 4 veranschaulicht den Ausdruck $\delta-\varphi$ aus (4.9) unter den Voraussetzungen der Abb. 2 und 3.

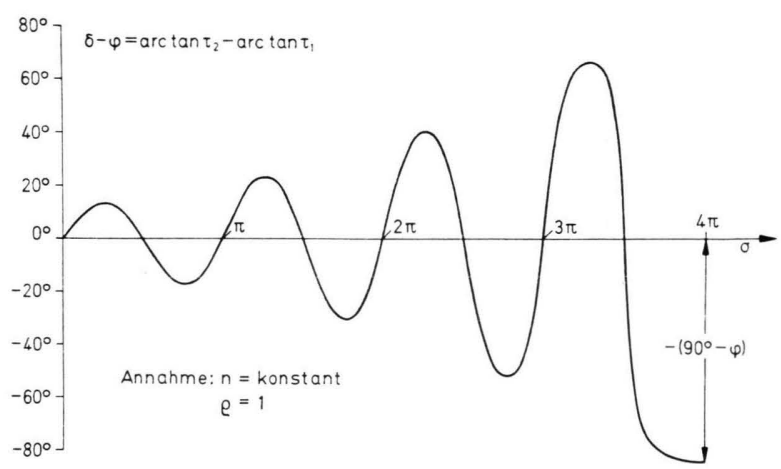

Abb. 4. Darstellung der Winkeldifferenz $\delta-\varphi$ einer partiellen stehenden Welle über der $\sigma$-Achse.

Die arc-Terme scheinen so lange schlecht zugänglich zu sein, wie der Ort $\sigma$ nicht meßtechnisch exponiert liegt.

Exponierte Orte sind $\sigma=0, \frac{\pi}{2}, \pi, \ldots$, solange diese Werte die Abszissenwerte der Wendepunkte der $(\delta-\varphi)$-Kurve darstellen.

Weiterhin heben sich die Orte $\sigma=\frac{\pi}{4}, \frac{3 \pi}{4}, \frac{5 \pi}{4}, \ldots$ hervor, welche den Abszissenwerten der Extrema der $(\delta-\varphi)$-Kurve gleich oder im allgemeinen wenigstens sehr nahe sind.

Für die genannten Fälle vereinfacht sich (4.9) wie folgt:

$$
\delta=\varphi \quad \text { für } \quad \sigma=0, \frac{\pi}{2}, \pi, \ldots
$$

und

$$
\begin{aligned}
& \delta=\varphi \pm\left(\frac{\pi}{2}-2 \arctan \frac{1-d}{1+d}\right) \\
& \text { für } \sigma=\frac{\pi}{4}, \frac{3 \pi}{4}, \frac{5 \pi}{4}, \ldots,
\end{aligned}
$$

wobei das Vorzeichen der Klammer in der Reihenfolge der genannten $\sigma$-Werte abwechselt.

Gleichung (4.10) hat den Vorzug größter Einfachheit. Entsprechend der Definition eines Wendepunktes sind die hierfür ausgesuchten Meßorte zugleich Orte größter Kurvensteilheit. Diese nimmt an den Orten der Gl. (4.10) mit wachsendem $\sigma$ zu (Abb. 4), was mit zunehmender experimenteller Unsicherheit gleichbedeutend ist. Daher sind die Meßorte dieses Falles nur bedingt brauchbar. Aus (4.9) gewinnt man für diese Stellen die Beziehung

$$
\frac{\mathrm{d} \delta}{\mathrm{d} \sigma}= \pm \frac{4 d}{1-d^{2}} .
$$

Die Meßgenauigkeit von $\delta$ ist dort besser oder gleich derjenigen von $\sigma$, solange $\mathrm{d} \delta / \mathrm{d} \sigma \leqq 1$, d. h., wenn $d \leqq 0,24$. Unter einer gleichwertigen Bedingung $\left(d^{2} \ll 1\right)$ kehrt der Wendepunkt zur $\sigma$-Achse und damit zu den Orten der Gl. (4.10) zurück.

Gleichung (4.11) macht die Kenntnis der Größe $d$ oder des Verhältnisses $v=(1-d) /(1+d)$ zur Ermittlung des Klammerausdruckes erforderlich. Wir setzen diesen vorläufig als bekannt voraus und berechnen analog zu (4.12)

$$
\frac{\mathrm{d} \delta}{\mathrm{d} \sigma}= \pm \frac{2 \cdot(\mathrm{d} d / \mathrm{d} \sigma)}{1+d^{2}}= \pm \frac{4 d \tan \varphi}{1+d^{2}} .
$$

Dieser Ausdruck läßt für alle $d$-Werte gute Meßgenauigkeit erwarten. Gl. (4.13) läßt beiläufig erkennen, daß die Orte der Gl. (4.11) und $\delta$-Extrema solange nicht zusammenfallen, wie nicht $d=$ konstant oder $\varphi=0$. Die Abweichung ist jedoch im allgemeinen gering und meßtechnisch ohne Bedeutung.

\subsection{Ermittlung des Verhältnisses $v=(1-d) /(1+d)$}

Die Gleichungen (4.3) und (4.11) machen die Bestimmung der Größe $v=(1-d) /(1+d)$ erforder. lich, um $\varphi$ am allgemeinen Ort bzw. an den Stellen $\sigma=\frac{\pi}{4}, \frac{3 \pi}{4}, \frac{5 \pi}{4}, \ldots$ ermitteln zu können. Es sei hier der Sonderfall $\sigma=0, \frac{\pi}{2}, \pi, \ldots$ betrachtet, für welchen (3.20) und (3.21) unter Benützung von (3.15) folgende Form annehmen:

$$
\frac{\left(E_{\mathrm{s}}|n|\right)_{\mathrm{e}}}{B_{1}}=\sqrt{1+d^{2} \pm 2} d=1 \pm d,
$$




$$
\frac{\left(H_{\mathrm{s}} Z_{0}\right)_{\mathrm{e}}}{B_{1}}=\sqrt{1+d^{2} \mp 2 d}=1 \mp d .
$$

Durch Division ergibt sich sofort:

$$
v=\frac{\left(H_{\mathrm{s}} Z_{0}\right)_{\mathrm{e}}}{\left(E_{\mathrm{s}}|n|\right)_{\mathrm{e}}} \quad \text { für die Orte } \sigma_{1}=0, \pi, 2 \pi, \ldots
$$

oder

$v=\frac{\left(E_{\mathrm{S}}|n|\right)_{\mathrm{e}}}{\left(H_{\mathrm{s}} Z_{0}\right)_{\mathrm{e}}}$ für die Orte $\sigma_{2}=\frac{\pi}{2}, \frac{3 \pi}{2}, \frac{5 \pi}{2}, \ldots$

Nach Vergleich mit Abb. 3 läßt sich folgende qualitative Formulierung gebrauchen: Man erhält $v$ dadurch, daß man die Minima der einen Komponente zu den entsprechenden Maxima der anderen Komponente ins Verhältnis setzt. - So gewinnt man $v$ im Abstand einer Viertelwellenlänge. Interpolation liefert $v$ für beliebige Orte. Unter Benützung von (4.5) lassen sich (4.16) und (4.17) allein durch Feldstärkewerte ausdrücken:

$v=\left[\frac{\left(H_{\mathrm{s}} Z_{0}\right)_{\mathrm{e}}}{\left(E_{\mathrm{s}}\right)_{\mathrm{e}}}\right]_{\sigma_{1}} \cdot \frac{\left(E_{\mathrm{s}}\right)_{\mathrm{e}}^{\text {extr }}}{\left(H_{\mathrm{S}} Z_{0}\right)_{\mathrm{e}}^{\text {extr }}}=\left[\frac{\left(E_{\mathrm{s}}\right)_{\mathrm{e}}}{\left(H_{\mathrm{S}} Z_{0}\right)_{\mathrm{e}}}\right]_{\sigma_{\mathrm{q}}} \cdot \frac{\left(H_{\mathrm{s}} Z_{0}\right)_{\mathrm{e}}^{\text {extr }}}{\left(E_{\mathrm{s}}\right)_{\mathrm{e}}^{\text {extr }}}$.

Sind einander entsprechende Maxima oder Minima örtlich gegeneinander versetzt, so findet man ihre Zentralwerte $\sigma_{1}$ und $\sigma_{2}$ auf Grund der beschriebenen Symmetrieverhältnisse (Abschnitt 4.2). Da die Enveloppen-Tangenten an den Stellen $\sigma_{1}$ und $\sigma_{2}$ im allgemeinen nur schwach oder vernachlässigbar geneigt sind, ist die Meßunsicherheit für $v$ gering.

\section{Zusammenfassung}

Die vorstehenden Betrachtungen zeigen, daß es auch beim Auftreten reflektierter Wellen möglich ist, den komplexen Brechungsindex eines Plasmas mit Hilfe des früher beschriebenen Ausbreitungsverfahrens zu ermitteln. Bei den Überlegungen wird von einer ebenen gedämpften elektromagnetischen Welle ausgegangen, die senkrecht reflektiert wird. Die resultierende partielle stehende Welle wird untersucht. Man gelangt zu folgendem Ergebnis:

Der Betrag des komplexen Brechungsindex läßt sich in einfacher Weise aus „Extremalkurven“ berechnen, welche im Experiment durch die Maxima bzw. Minima (Täler) der Enveloppen beider Feldstärkekomponenten markiert sind. Das Argument des komplexen Brechungsindex ergibt sich aus dem Phasenunterschied beider Komponenten, der an meßtechnisch geeigneten Orten mit einem Abstand bis zu einer Viertelwellenlänge gemessen wird; gegebenenfalls werden auch noch die Amplituden beider Komponenten jeweils am Ort ihrer Extrema verglichen.

Die unmittelbar anfallende, diskrete Folge von Meßpunkten schränkt das örtliche Auflösungsvermögen des Verfahrens nicht ein, da das Medium als langsam veränderlich vorausgesetzt war. 